\title{
Few-electron semiconductor quantum dots with Gaussian confinement
}

\author{
Sergio S. Gomez ${ }^{12 *}$, Rodolfo H. Romero ${ }^{12 \dagger}$ \\ 1 Facultad de Ciencias Exactas y Naturales, Universidad Nacional del Nordeste, \\ Avenida Libertad 5500, (3400) Corrientes, Argentina \\ 2 Instituto de Modelado e Innovación Tecnológica, CONICET, \\ Avenida Libertad 5500, (3400) Corrientes, Argentina
}

Received 18 June 2008; accepted 23 September 2008

\begin{abstract}
:
We have performed Hartree-Fock calculations of the electronic structure of $N \leq 10$ electrons in a quantum dot modeled with a confining Gaussian potential well. We discuss the conditions for the stability of $N$ bound electrons in the system. We show that the most relevant parameter determining the number of bound electrons is $V_{0} R^{2}$. Such a feature arises from widely valid scaling properties of the confining potential. Gaussian Quantum dots having $N=2,5$, and 8 electrons are particularly stable in agreement with the Hund rule. The shell structure becomes less and less noticeable as the well radius increases.
\end{abstract}

PACS (2008): 73.21.-b; 73.22-f

Keywords: $\quad$ Quantum dots $•$ Gaussian potential $•$ electronic structure

(c) Versita Warsaw and Springer-Verlag Berlin Heidelberg.

\section{Introduction}

Modern semiconductor technology has allowed the fabrication and manipulation of electrons confined within regions of nanometer size having a plethora of shapes. The possibility of tuning the shape, size, and number of bound electrons of those nanostructures has raised a lot of interest in the subject of confined low-dimensional few-electron systems known as quantum dots (QDs) [1, 2] either for specific applications or for exploring new fundamental phenomena at the quantum level [3-5]. At present, much

*E-mail: ssgomez@exa.unne.edu.ar

†E-mail: rhromero@exa.unne.edu.ar work is being performed on optical investigations of single and coupled dots as a first step towards solid-state-based quantum communication and computing devices [6].

Quantum dots have also been termed "artificial atoms" because their electronic structure and properties resemble those of natural atoms [7]. Electrons in quantum dots are confined due to potential barriers in much the same way as electrons in atoms are confined due to the Coulomb attraction of the nucleus. Further similarities arise because electrons within quantum dots interact with each other through Coulomb forces; their energy spectra present both discrete and continuum states giving rise to binding and dissociation processes, while transitions between them give rise to emission and absorption of radiation.

Thus, it is justified, to some extent, to apply-mutatis mutandis-methods of atomic or molecular physics to the 
study of these systems of electrons subjected to a given confining potential. A spherically symmetric confining potential can provide a model for semiconductor spherical nanocrystals embedded within an insulator [8]. In the literature, calculations of the electronic structure and the properties of QDs are often performed with a confining harmonic-potential model. This can be justified on the basis of a generalization of Kohn's theorem [9] satisfied by such a potential and, approximately, verified by experiments. A potential of this type allows the explanation of the observed electronic shell structure, e.g., in small clusters. Nevertheless, the infinite depth and range of a parabolic potential is clearly unphysical: it can accommodate an infinite number of bound electrons, which precludes the consideration of binding and dissociation processes.

Finite-range models have been also advocated, e.g., the energy spectra of two- and three-electron systems in a spherical potential well of finite depth was obtained variationally [10], and the unrestricted Hartree-Fock (UHF) method was applied to the same model for the calculation of the electronic structure of systems having up to 20 electrons [11]. More recently, a UHF calculation of ground state, chemical potential, and charging energies of electrons in an infinite spherical potential well, with and without magnetic field, has been reported [12]. However, the sharp discontinuity at the QD radius for such a potential well is not completely satisfactory both from a physical and a computational point of view, and some interpolating potentials [13] as well as smoothly varying potentials have been proposed [14-18]. Among them, the Gaussian potential has received some attention and its one- [19-22] and two-electron spectra [15-17] have been calculated.

In self-assembled QDs, a small interdiffusion between the dots and the surrounding materials is expected to produce a significant change in the band structure and the optical properties. Therefore, smooth potentials such as Gaussian ones, could be useful, for instance, in qualitatively describing this type of effects [23]. Furthermore, it is particularly suitable for an atomic-like treatment, such as UHF calculations. The Hartree-Fock method is one of the most widely used methods for the calculation of the electronic structure of atoms and molecules. Its usual implementation consists in the expansion of the unknown orbitals as a linear combination in a given basis set. The method thus determines self-consistently the energy eigenvalues and the orbitals.

In the Hartree-Fock approach, the electron-electron correlation is neglected. In Refs. [10, 11], a detailed study was performed of the problem of correlation for manyelectron artificial atoms in a spherical QD with a finite confinement potential. In those references, it was shown that the Hartree-Fock and exact results are almost identical for QDs with small and intermediate radii, such as those considered in the present work. On the other hand, such a methodology is the starting point for a systematic improvement in the treatment of correlation effects by, e.g., higher-order perturbation theory or multi-reference methods $[24,25]$.

In atomic problems, the most common basis sets are spherical or Cartesian Gaussian functions because they provide closed expressions for the matrix elements of the atomic Hamiltonian. Such an advantage applies even more when the confining potential is Gaussian itself. Hence, we take in this work an atomic-like approach for the calculation of the electronic structure of few-electron Gaussian quantum dots. Such an approach shall be particularly advantageous when dealing with, e.g., systems of coupled QDs, and also for the analysis of mixed systems, like molecules coupled to QDs. Work along this line will be published elsewhere. We also disregard strain effects throughout this work, although they can be important in self-assembled QDs [26]. This approximation would be justified as long as the mismatch between the substrate and QD lattice constants is small.

\section{Theory}

We consider a system of few electrons confined by a potential assumed to be a spherically symmetric Gaussian potential well of typical radius $R$ and finite depth $-V_{0}$, i.e., $V(r)=-V_{0} \exp \left(-r^{2} / 2 R^{2}\right)$. It approaches a parabolic behavior around its minimum while going smoothly to zero at infinity. In Ref. [15], the parabolic approximation

$$
V(r)=-V_{0} e^{-r^{2} / 2 R^{2}} \simeq-V_{0}+V_{0} \frac{r^{2}}{2 R^{2}}=-V_{0}+\frac{1}{2} m \omega^{2} r^{2}
$$

was considered for comparison, where

$$
\omega^{2}=\frac{V_{0}}{m R^{2}}=\frac{2 \lambda V_{0}}{m}
$$

relates the frequency $\omega$ of the harmonic oscillator to the Gaussian exponent $\lambda \equiv 1 / 2 R^{2}$. The Schrödinger equation for a system of $N$ electrons confined within a Gaussian well and interacting through Coulomb potentials is

$$
\left[-\frac{\hbar^{2}}{2 m^{*}} \sum_{i} \nabla_{i}^{2}+\sum_{i} V\left(r_{i}\right)+\sum_{i<j} \frac{e^{2}}{4 \pi \kappa \epsilon_{0} r_{i j}}\right] \psi=E \psi,
$$

where $m^{*}$ is the effective electron mass, and $\kappa=\epsilon / \epsilon_{0}$ is the dielectric constant of the medium. We neglect the 
changes of the effective electron mass and the dielectric constant at the QD boundary. By introducing the donor Bohr radius $a_{D}=\left(\mathrm{km} / \mathrm{m}^{*}\right) a_{B}$ as the unit of length and the donor Rydberg $R_{D}=\left(m^{*} / m \kappa^{2}\right)$ Ry as the unit of energy, Eq. (3) becomes

$$
\left[-\sum_{i} \nabla_{i}^{2}+\sum_{i} V\left(r_{i}\right)+\sum_{i<j} \frac{2}{r_{i j}}\right] \psi=\varepsilon \psi,
$$

so the results can be easily transferred between different materials by properly changing $a_{D}$ and $R_{D}$; for instance, for a GaAs semiconductor $\mathrm{QD}$, one has $R_{D}=6 \mathrm{meV}$ and $a_{D}=10 \mathrm{~nm}$.

The existence of bound states in the system described by Eq. (4) depends on the radius and depth of the Gaussian potential. If both magnitudes are small enough, not even a single electron can form a stable bound state. Some physical insight on the stability of one- and few-electron QDs can be gained from a simple variational estimate. The discussion will also prove to provide a useful starting point for the systematic construction of basis sets used in the more complicated many-electron calculations reported below. Let $E_{0}^{(N)}$ be the ground-state energy of an $N$ electron QD. Let us estimate variationally the ground state energy of one electron in a Gaussian potential. Taking into account that, around its center, the Gaussian potential resembles a parabolic one, we propose a normalized $s$ type Gaussian trial function of exponent $\alpha$

$$
\varphi_{s}(r)=(2 \alpha / \pi)^{3 / 4} \exp \left(-\alpha r^{2}\right)
$$

The ground state in the well becomes increasingly similar to that of the harmonic oscillator when the well depth is growing. The expectation value of the one-electron Hamiltonian then becomes

$$
E(\alpha)=3 \alpha-V_{0}\left(\frac{2 \alpha}{\lambda+2 \alpha}\right)^{3 / 2}
$$

where the first and second terms are the mean values of the kinetic energy and the confining potential, respectively. Minimization with respect to $\alpha$, i.e., $\partial E(\alpha) / \partial \alpha=0$, gives

$$
(\lambda+2 \alpha)^{5}-8 \lambda^{2} V_{0}^{2} \alpha=0,
$$

which has to be solved numerically for given $\lambda$ and $V_{0}$. Equation (7) has five roots but, typically, the one that minimizes $E(\alpha)$, is a positive number of order unity $\alpha=\alpha_{\text {opt }}$. The other roots can be rejected on physical grounds: they are either complex conjugate pairs, or real but negative, or too close to zero, and the corresponding trial functions therefore do not describe bound states. Thus, within this variational approach, the energy of a single-electron QD is

$$
E_{0}^{(1)}=E\left(\alpha_{\mathrm{opt}}\right)
$$

On the other hand, the ground state of a two-electron QD corresponds to one electron with spin $+\frac{1}{2}$ and the other with spin $-\frac{1}{2}$ interacting with each other through the Coulomb interaction $V_{e e}=2 / r_{12}$. Therefore,

$$
E^{(2)}=2 E_{0}^{(1)}+J
$$

where $J$ is the energy shift of the one-electron levels due to the Coulomb interaction between two Gaussian charge densities

$$
J=2 \int\left|\varphi_{s}\left(\mathbf{r}_{1}\right)\right|^{2} \frac{1}{r_{12}}\left|\varphi_{s}\left(\mathbf{r}_{2}\right)\right|^{2} d^{3} \mathbf{r}_{1} d^{3} \mathbf{r}_{2}=4 \sqrt{\frac{\alpha_{\mathrm{opt}}}{\pi}} .
$$

The condition of stability of the two-electron QD discussed in Refs. [10, 11] and applied in Refs. [15, 17], $E_{0}^{(2)}<E_{0}^{(1)}$, is equivalent to

$$
E_{0}^{(1)}<-J
$$

We shall give a more thorough discussion of the conditions for stability when we consider the results for few-electron QDs.

The calculations reported in this work have considered both one- and few-electron systems. The former were used for benchmark purposes and provide a systematic way for calibrating the method. A one-electron Gaussian potential of $V_{0}=400 R_{D}$ and $\lambda=1$ has been considered by various authors for comparing methods proposed in the literature. We also address such a case as our first calculation to assess the accuracy of our methodology. The first issue to be considered is the choice of a suitable Cartesian Gaussian basis set $\left\{\varphi_{\ell}^{(i)}\right\},(i=1, \ldots, K)$, where

$$
\varphi_{\ell}^{(i)}=x^{m} y^{n} z^{p} \exp \left(-\alpha_{i} r^{2}\right)
$$

and $\ell=m+n+p$ is the angular momentum of the function. Hereafter we use the spectroscopic notation $s, p, d, \ldots$ for $\ell=0,1,2, \ldots$, i.e.,

$$
\left\{\varphi_{\ell}^{(i)}\right\}=\left\{\varphi_{s}^{(i)}, \varphi_{p_{x}}^{(i)}, \varphi_{p_{y}}^{(i)}, \varphi_{p_{z}}^{(i)}, \varphi_{d_{x x}}^{(i)}, \varphi_{d_{x y}}^{(i)}, \ldots\right\}
$$

The one-electron QDs were solved by direct diagonalization of the Hamiltonian, while the many-electron systems were treated with the UHF method. The calculations for a given potential, i.e., for a given pair $\left(V_{0}, \lambda\right)$, were performed 
Table 1. Values of the parameters $\lambda$ and $V_{0}$ for the Gaussian potentials studied. The radii of the quantum dots are given by $R=1 / \sqrt{2 \lambda}$. The quantities $\alpha_{\mathrm{opt}}$ are the variationally optimized exponents used to construct the basis sets.

\begin{tabular}{cccc}
\hline \hline$\lambda$ (a.u. $\left.^{*}\right)$ & $V_{0}\left(R_{D}\right)$ & $R\left(a_{D}\right)$ & $\alpha_{\text {opt }}\left(a_{D}^{-2}\right)$ \\
\hline 1.0 & 400 & 0.707 & 9.370885 \\
0.5 & 50 & 1.0 & 2.183148 \\
0.5 & 15 & 1.0 & 1.047992 \\
\hline \hline
\end{tabular}

with the same basis sets, irrespective of the number of electrons.

The prescription for the choice of the basis is based on the previously discussed variational energy expression (6) and the optimal exponent $\alpha_{\text {opt }}$ defined by Eq. (7). The values of $\alpha_{\text {opt }}$ for the three cases considered in this paper are listed in Table 1. Those exponents were then used to generate the basis functions with higher angular momentum, i.e., we chose $\alpha_{s}=\alpha_{p}=\alpha_{d}=\ldots=\alpha_{\mathrm{opt}}$. This procedure can be justified as long as the Gaussian potential is similar to the parabolic potential, whose solutions are Hermite polynomials multiplied by Gaussian functions with an exponent independent of the principal and angular quantum numbers $n$ and $\ell$, which satisfies exactly the prescription given above.

By including one Gaussian function with the optimized exponent in each angular-momentum block up to a maximum value $L$, i.e., $0 \leq \ell \leq L$, the low-lying states are reasonably well reproduced as compared to previously reported values. In particular, the ground-state energy is approximately correct to within $10^{-2} R_{D}$, as expected, because of the variational procedure for obtaining the basis exponents.

Generally, states having $\ell>L$ are missing or, if obtained, have larger errors. This is also a consequence of the fact that the low-lying states of the parabolic and Gaussian potentials are alike, but those of high angular momentum are not. Hence, to get a correct description of high-lying excited states, functions with the corresponding angular momentum $\ell$ have to be included. In atomic and molecular calculations, a useful procedure for enlarging the basis set has been the so-called even-tempered criterion [27] by which a basis set containing various functions of the same angular momentum have their exponents in the same ratio, i.e., for a given $\ell$, the exponents $\alpha_{\ell}^{(1)}, \alpha_{\ell}^{(2)}, \alpha_{\ell}^{(3)}, \ldots$ are in the ratio $\alpha_{\ell}^{(i+1)} / \alpha_{\ell}^{(i)}=$ const. In the limit of a large number of functions, the basis set should become complete, independently of the ratio chosen. We arbitrarily take a ratio of two to enlarge every block of angular momentum in the original basis set, thus including exponents $\alpha_{\ell}^{(i)}=\alpha_{\mathrm{opt}} / 2^{i-1}$ $(i=1, \ldots, K)$ smaller than the optimized one. A value of $K=4$ has been enough to reach convergence in energy to within $10^{-3} R_{D}$ for all the calculations reported here. In summary, the basis set used in all calculations consists of a set $\left\{\varphi_{s}^{(i)}, \varphi_{p}^{(i)}, \ldots, \varphi_{\ell}^{(i)}, \ldots\right\}$, with $i=1, \ldots, K=4$ and $\ell \leq L=4$, having 140 Cartesian Gaussian functions.

\section{Results and discussion}

\subsection{One-electron quantum dots}

In Table 2, we have listed the energy eigenvalues calculated for the potentials studied. The first potential, $V_{0}=400$, has been investigated previously [19-22] and provides a measure for the precision of our calculations. The first column refers to energies calculated by diagonalizing the matrix representation of the Hamiltonian after expansion in the basis set described above. The second column corresponds to a numerical solution of the Schrödinger equation by using Numerov's integration algorithm [28], which we have implemented as an independent verification for the basis-set expansion method. The third column compares our results for the $V_{0}=400$ potential to previous calculations available in the literature; in particular, the values from Ref. [20] are listed, which are probably the most accurate results reported so far. This potential has bound states with angular momenta as high as $\ell=7$, which makes it difficult to reproduce with a basis set having functions with $\ell=0$ through $\ell=4$ only. This is particularly apparent for the energies $E_{2 f}$ and $E_{2 g}$, having the largest errors. This effect of the incompleteness of the basis set can be removed by adding four extra functions for each $\ell$ value and symmetry with $5 \leq \ell \leq 7$, which amounts to a total of 360 basis functions. The energies calculated with this enlarged basis become $E_{2 f}=-170.639 R_{D}$ and $E_{2 g}=-140.133 R_{D}$, in good agreement with Ref. [20]. Nevertheless, the need for these more demanding calculations may only arise when those states are occupied or in the presence of perturbations exciting such states. 
Table 2. Bound-state energies of the one-electron spherical Gaussian potentials $\left(V_{0}, \lambda\right)=(400,1),(50,0.5)$, and $(15,0.5)$ calculated by diagonalization of the Hamiltonian matrix and Numerov's integration method. Energies are given in donor Rydberg units $R_{D}$ and lengths in donor Bohr radius $a_{D}$, and dots stand for positive-energy states.

\begin{tabular}{|c|c|c|c|c|c|c|c|}
\hline \multirow[t]{2}{*}{$n \ell$} & \multicolumn{3}{|c|}{$V_{0}=400 \mathrm{R}_{D}$} & \multicolumn{2}{|c|}{$V_{0}=50 \mathrm{R}_{D}$} & \multicolumn{2}{|c|}{$V_{0}=15 \mathrm{R}_{D}$} \\
\hline & Diagon. & Numerov & Ref. [20] & Diagon. & Numerov & Diagon. & Numerov \\
\hline $1 \mathrm{~s}$ & -341.895 & -341.892 & -341.8952 & -35.958 & -35.958 & -7.762 & -7.762 \\
\hline $1 p$ & -304.463 & -304.463 & -304.4628 & -27.282 & -27.282 & -3.697 & -3.698 \\
\hline $2 s$ & -269.644 & -269.640 & -269.6445 & -19.987 & -19.987 & -1.215 & -1.215 \\
\hline $1 d$ & -268.110 & -268.111 & -268.1107 & -19.204 & -19.204 & -0.439 & -0.439 \\
\hline $2 p$ & -235.446 & -235.450 & -235.4500 & -13.109 & -13.111 & & \\
\hline $1 f$ & -232.849 & -232.875 & -232.8753 & -11.780 & -11.785 & & \\
\hline $3 s$ & -203.983 & -203.979 & -203.9835 & -7.800 & -7.800 & & \\
\hline $2 d$ & -202.427 & -202.431 & -202.4313 & -7.009 & -7.010 & & \\
\hline $1 g$ & -198.700 & -198.798 & -198.7983 & -5.111 & -5.122 & & \\
\hline $3 p$ & -173.156 & -173.244 & -173.2443 & -3.173 & -3.179 & & \\
\hline $2 f$ & -167.797 & -170.639 & -170.6393 & -1.864 & -1.876 & & \\
\hline $4 s$ & -145.372 & -145.373 & -145.3779 & -0.598 & -0.600 & & \\
\hline $3 d$ & -143.741 & -143.809 & -143.8091 & 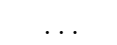 & & & \\
\hline $2 g$ & -138.045 & -140.135 & -140.1351 & $\ldots$ & $\ldots$ & & \\
\hline
\end{tabular}

The case $V_{0}=50 R_{D}$ shows a good agreement between direct diagonalization and numerical integration implying that both methods and the basis set used are accurate enough. The scheme of levels obtained is in agreement with that in Ref. [15]. The system $V_{0}=15 R_{D}$ has not been treated previously, and it provides a system having just a few bound states. In this situation, the parabolic approximation gives probably poor results.

It can also be seen from Table 2 that the energy of the corresponding parabolic potential, $\hbar \omega=\sqrt{2 \lambda V_{0} / m}=$ $5.48,10$, and $40 R_{D}$, for $V_{0}=15,50$, and $400 R_{D}$ respectively, represents approximately the energy difference between the ground state and the first excited state. If the potential were really parabolic, that energy difference would be the same for every pair of consecutive states. That is not the case for the Gaussian potential, i.e., the higher the pair of states considered lie, the worse the approximation becomes, as already noted by other authors $[15,17]$. The basis sets optimized for these one-electron calculations will now be used for few-electrons systems.

\subsection{Few-electron quantum dots}

The results of our Hartree-Fock calculations of the ground-state energy of $N$-electron systems $(N=$ $1, \ldots, 10)$, as a function of the QD radius, for $V_{0}=15 R_{D}$ (top panel) and $V_{0}=50 R_{D}$ (bottom panel), are shown in Fig. 1. This figure has features similar to those obtained with a finite-depth square well [11]. For comparison, we have also plotted (dashed lines) the variational one- and two-electron energies $E_{0}^{(1)}$ and $E_{0}^{(2)}$, given by Eqs. (6) and(9), respectively.

The figure shows several critical radii $R_{c}^{(N)}$ at which a crossover between the $E_{0}^{(N)}$ and $E_{0}^{(N-1)}$ ground states occurs. Thus, $R_{c}^{(N)}$ is the minimum radius to have $N$ bound electrons; for $R>R_{c}^{(N)}$, the $N$-electron QD becomes more stable than the $(N-1)$-electron one. It should be noted that at small enough radii no bound state exists. At radii less than $R_{c}^{(2)}=0.43 a_{D}$ (for $V_{0}=15 R_{D}$ ) and $R_{c}^{(2)}=0.23 a_{D}$ (for $R=50 R_{D}$ ), only a single electron can be bound, but for $R>R_{c}^{(2)}$ we have $E_{0}^{(2)}<E_{0}^{(1)}$. The radii for $N=1$ and $N=2$ are quite similar $\left(R_{c}^{(1)} \simeq R_{c}^{(2)}\right)$. This also occurs for $R_{c}^{(3)} \simeq R_{c}^{(4)} \simeq \ldots \simeq R_{c}^{(8)}$ and $R_{c}^{(9)} \simeq R_{c}^{(10)}$.

Most of the general features of the stability of the $N_{-}$ electron system can be understood considering the oneelectron energies. An electron confined within a well with a typical size $R$ will have a momentum of order $\hbar / R$ due to the uncertainty principle; its kinetic energy is then of or$\operatorname{der} \hbar^{2} / 2 m R^{2}$, which approaches zero as $R$ goes to infinity. On the other hand, its potential energy $-V_{0} \exp \left(-r^{2} / 2 R^{2}\right)$ approaches the bottom of the well $-V_{0}$. Then, in the limit of large radius, the energy of the one-electron system goes to $-V_{0}$ and, since also the Coulomb interaction goes to zero, the energy of the $N$-electron well approaches $-N V_{0}$. 

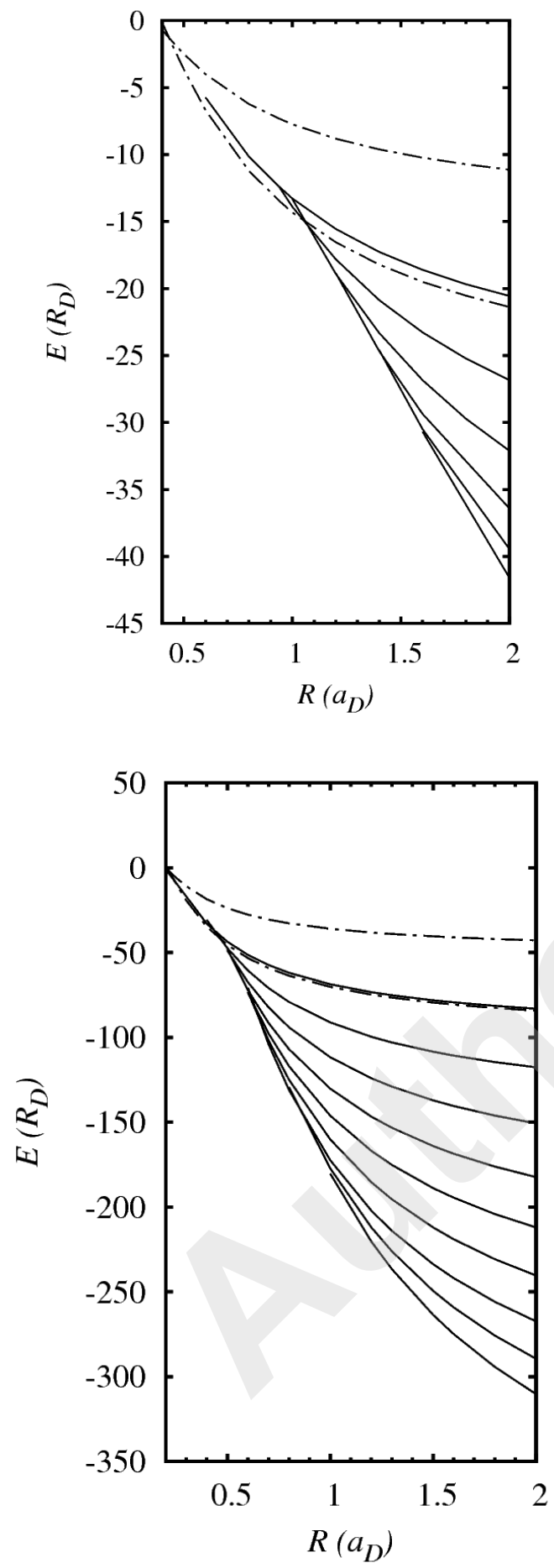

Figure 1. Ground-state energies $E_{0}^{(N)}$ of $N$-electron Gaussian quantum dots $(N \leq 10)$ as a function of the dot radii for the depths $V_{0}=15 R_{D}$ (top) and $V_{0}=50 R_{D}$ (bottom). The cross-over of levels occurs when $E_{0}^{(N+1)} \leq E_{0}^{(N)}$ at a critical radius $R_{c}^{(\mathcal{N})}$, starting from approximately $R_{c}^{(1)}=0.43 a_{D}$ and $R_{c}^{(1)}=0.22 a_{D}$, respectively.
Such a trend can be observed in Fig. 1.

At a given finite radius, however, the energies $E_{0}^{(N)}$ are not equally spaced for successive $N$, because of the shell structure of the energy levels in the potential well. This shell structure is even more strikingly revealed in the top panel of Fig. 2, where the chemical potential $\mu(N)$ is depicted as a function of the QD radius. The chemical potential represents the affinity of the well for binding an extra electron. It is the equivalent of the ionization potential or the electron affinity in atomic physics. A grouping of the lines corresponding to the number of electrons occupying the same one-electron level is clearly apparent. All curves decrease as $R$ increases due to the fact that when the electrons becomes less confined, the system approaches a classical behavior and it is easier to add a new electron. In the bottom panel of Fig. 2, the charging energy, defined as the difference between the chemical potential of two systems differing in one electron, i.e. $E_{\text {char }}^{(N)}=\mu(N+1)-\mu(N)$, is depicted as a function of the number of electrons in the QD for three potential radii, namely, $0.8,1.5$ and $2 a_{D}$. The charging energy gives a measure of the stability of the system, the larger $E_{c h a r}$, the more stable the system. For $N=2,5$, and 8 electrons, the system presents large values of $E_{c h a r}$, in correspondence with the number of electrons needed for filling or half-filling a shell. The peak heights also diminish as the QD radius increases. That is, the charging energy as a function of $N$ tends to be flat because of the disappearance of the shell structure in this classical limit.

As an illustration of the relation between the QD depth and the critical radius, we have depicted, in Fig. 3, the curves $V_{0}$ versus $1 / R_{c}^{2}$ for a QD of three interacting (upper curve) and non-interacting electrons (lower curve). They are the locus of the points representing the minimum QD radii for a given depth. Both curves are nearly straight lines, thus showing that $V_{0} R_{c}^{2}$ is approximately constant. We shall show, in the following, that the variational energy of the one-electron Gaussian potential also has such a property. By defining the dimensionless variable $x=2 \alpha / \lambda$, the energy $E_{0}(\alpha)$ defined by Eq. (6) can be written as

$$
E(\alpha)=\lambda\left[\frac{3}{2} x-2 C\left(\frac{x}{x+1}\right)^{3 / 2}\right]=\lambda \varepsilon(C),
$$

where $C=V_{0} R^{2}$ and $\varepsilon$ is the dimensionless energy depending on $C$. On the other hand, the equation determining the optimal variational exponent can be written as

$$
\frac{\partial \varepsilon}{\partial x}=\frac{3}{2}-3 C \frac{x^{1 / 2}}{(x+1)^{5 / 2}}=0 .
$$

The equation $\varepsilon(C)=0$ represents the condition to have at least one bound state, which clearly only depends on 

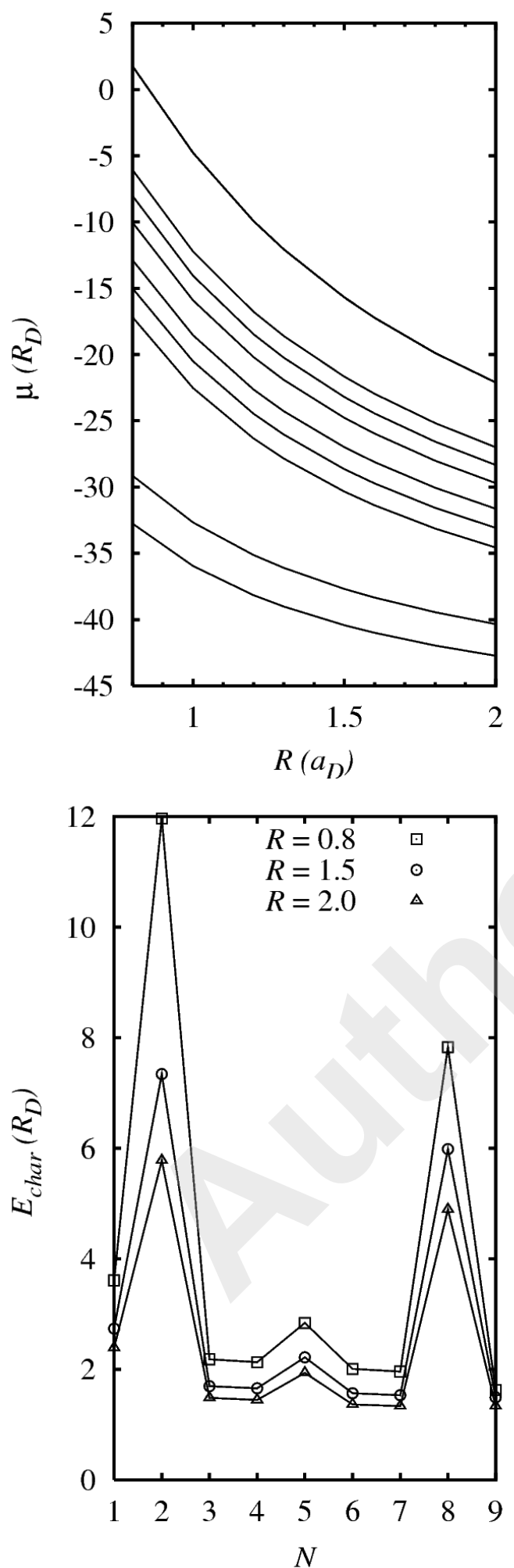

Figure 2. Chemical potential (top) and charging energy (bottom) of the $\mathrm{N}$-electron Gaussian potentials considered in this work. The grouping of lines in the chemical potential $\mu$ and the peaks in the charging energy show the shell structure.

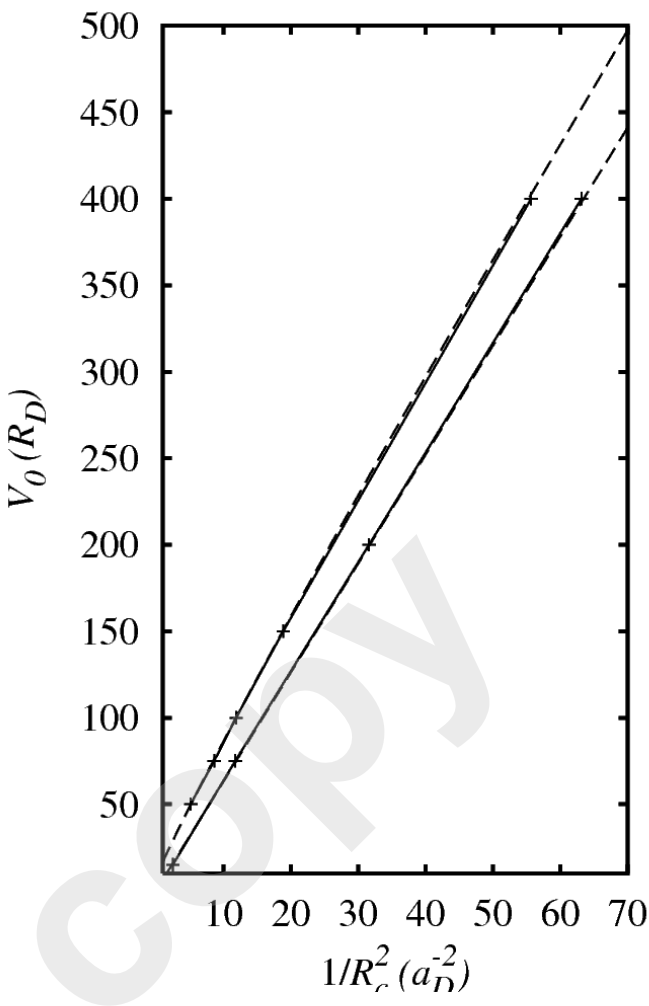

Figure 3. Critical lines representing the relation between the potential depth $V_{0}$ and $1 /\left[R_{c}^{(3)}\right]^{2}$, where $R_{c}^{(3)}$ is the minimal radius to have three bound electrons in the system. The lower curve represents the critical line for the non-interacting problem and its fit to the straight line $V_{0}=6.3 R^{-2}$. The upper curve represents the critical UHF line. The dashed line corresponds to the fit $V_{0}=7.4+9.0 R^{-1.88}$.

$V_{0} R^{2}$. This equation and Eq. (15) are simultaneously satisfied if $x=1 / 2$ and $C=9 \sqrt{3} / 8$, i.e., $\alpha=\lambda / 4=1 / 8 R^{2}$ and $V_{0} R^{2}=1.95$. Hence, for a given $V_{0}$, the minimal radius for having one bound electron is $R_{c}^{(1)}=\sqrt{1.95 / V_{0}}=$ $1.40 / \sqrt{V_{0}}$.

This can be compared to the results we would obtain with the truncated parabolic approximation of the Gaussian potential, i.e., $V(r)=-V_{0}+m \omega^{2} r^{2} / 2$ if $r<R$, and zero otherwise, with $\omega$ given by Eq. (2). In such a case, the condition for having at least one bound state is that the zero-point energy becomes less than or equal to zero. The critical radius is therefore determined by

$$
E_{0}^{(1)}=-V_{0}+\frac{3}{2} \hbar \omega=-V_{0}+\frac{3}{2} \hbar \sqrt{\frac{V_{0}}{m R^{2}}}=0
$$

This gives explicitly $V_{0} R_{c}^{2}=9 / 4=2.25$, i.e., $R_{c}^{(1)}=$ $1.5 / \sqrt{V_{0}}$, which is comparable to the relation obtained above. 
If there were no interactions between the electrons, the energy for allocating the second electron would be the same, because both of them would occupy the one-electron ground state, although with opposite spins. The Coulomb interaction between the electron pair, however, modifies such an energy by an amount $J$. Nevertheless, $J$ is, for small- and medium-size QDs, much smaller than the energy difference between the ground state and the first excited one-electron states. Thus, the next critical radius $R_{c}^{(2)}$ is near to the first one $R_{c}^{(1)}$. On the other hand, the radius $R_{c}^{(3)}$ includes the effects of both the electronelectron interaction and the shell structure because the third electron has to occupy the first excited one-electron level. We can estimate this radius by using an argument similar to the one above. The first excited state of the harmonic oscillator is now (5/2) $\hbar \omega$ above the bottom of the well, thus giving $V_{0} R_{c}^{2}=25 / 4=6.25$. A fit of the plot in Fig. 3 of the form $V_{0}=a+b R_{c}^{\gamma}$ gives $V_{0}=6.30 R_{c}^{-2}$ for the non-interacting case, which compares fairly well to the estimation from the truncated harmonic oscillator, thus showing that the non-interacting picture is qualitatively correct. Nevertheless, the corresponding fit of the UHF calculations gives a relation $V_{0}=7.4+9.0 R^{-1.88}$ as a consequence of the Coulomb and exchange interactions. Furthermore, the fact that $V_{0} R^{2}$ determines the number of bound electrons is a property shared by numerous types of potentials. Let $H$ be a Hamiltonian of the form

$$
H=-\nabla^{2}-V_{0} u(\rho)
$$

where the potential can be factored as a product of a typical energy $-V_{0}$ times a function $u(\rho)$ of the dimensionless variable $\rho=r / R$. This includes most of the types of confining potential used in previous works, e.g., the square well $V(r)=-V_{0} \theta(1-\rho)$, the parabolic potential $V(r)=\hbar \omega(r / R)^{2}$ with $R=\sqrt{\hbar / 2 m \omega}$ and, clearly, the Gaussian potential. Expressing the Laplacian in terms of $\rho$, we get

$$
H=\frac{1}{R^{2}}\left[-\nabla_{\rho}^{2}-V_{0} R^{2} u(\rho)\right],
$$

which has energy eigenvalues $\varepsilon_{n}\left(V_{0}, R\right)=\epsilon_{n}\left(V_{0} R^{2}\right) / R^{2}$. Neglecting the electron-electron interaction, the condition for stability of an $N$-electron system is just $\varepsilon_{n}<0$, where $n$ is the quantum number corresponding to the highest occupied state. It is fulfilled for certain values of $V_{0} R_{c}^{2}=C_{i}$ giving rise to stability regions.

Therefore, when the electron-electron interaction $V_{e e}$ can be neglected, the plot of $V_{0}$ versus $1 / R_{c}^{2}$ represents a phase diagram consisting of regions delimited by straight lines determining the various zones where $N$ electrons can form a bound state. The argument does not hold when $V_{e e}$ is not negligible, since the kinetic and the $V_{e e}$ term scale differently with $R$.
Table 3. Electronic configuration and $z$ projection of the total spin $M_{S}$ for the most stable UHF configurations of an $\mathrm{N}$-electron Gaussian potential of $V_{0}=50 R_{D}$, calculated in the present work. The spin projections of the orbital are represented by $\uparrow$ and $\downarrow$, for $+1 / 2$ and $-1 / 2$, respectively. Spin indices are omitted for closed-shell configurations. Hund's Rule is satisfied for all configurations shown.

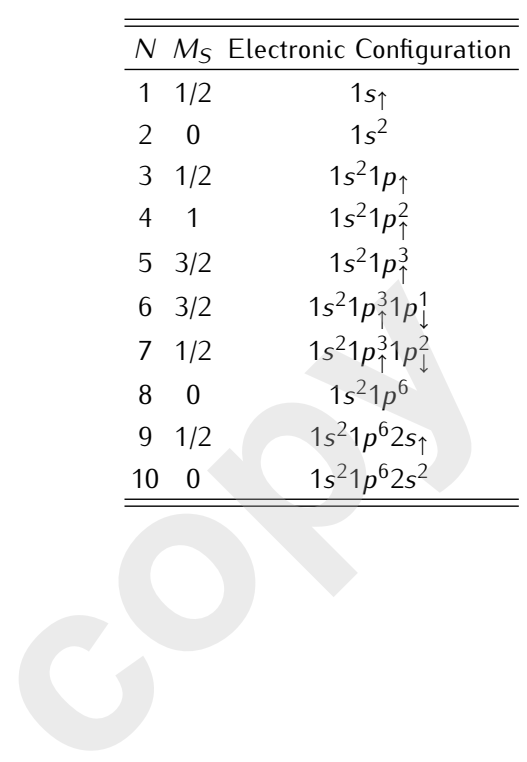

It is interesting to analyze how the electrons occupy the UHF spin orbitals as they are added into the system. Table 3 shows the most stable UHF configurations, for a given $N$, along with the total spin projection $M_{S}$, for $V_{0}=50 R_{D}$ and $R \geq R_{c}^{(10)}$. In all the calculations performed, with $N \leq 10$, Hund's rule is satisfied, i.e., electrons in the same shell maximize the projection of the total electronic spin, in agreement with previous works. As an example, we consider the UHF levels of a $V_{0}=15 R_{D}$ well of $R=1 a_{D}$. Figure 4 shows how the scheme of levels changes as the first three electrons are added to it. Panel (b) shows the one-electron levels of the potential with a single electron occupying the ground state $1 \mathrm{~s}$. When a second electron is added to the system, the pair can form a state with total spin projection $M_{S}=1$ [panel (a)] or $M_{S}=0$ [panel (c)]. Panels (a) and (d) show the states available for spins $+\frac{1}{2}$ and $-\frac{1}{2}$ drawn adjacent to each other. The electron-electron repulsion shifts and splits the one-electron levels through the Coulomb and exchange interactions. In the $M_{S}=0$ configuration, both electrons can occupy the same orbital, while the $M_{S}=1$ configuration requires the second electron to occupy the higher $p_{\uparrow}$ orbital, thus giving a higher total energy. The effect of adding a third electron to the $M_{S}=0$ configuration is shown in panel (d). The $1 s$ orbital shifts upwards and splits into $1 s_{\uparrow}$ and $1 s_{\downarrow}$, while the $1 p_{\uparrow}$ becomes stabilized. 


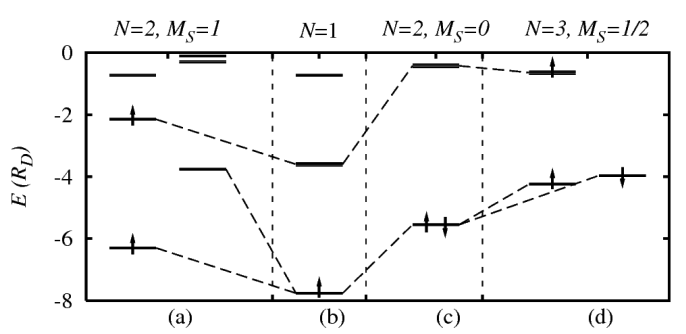

Figure 4. Scheme of the UHF levels for the $V_{0}=15 R_{D}$ and $R=1 a_{D}$ Gaussian well having one, two, and three bound electrons. The notation $\uparrow$ and $\downarrow$ refers to the spin projections. In the cases $N=2, M_{s}=1$ [panel (a)] and $N=3, M_{s}=1 / 2$ [panel (d)], the states for spins $\uparrow$ and $\downarrow$ are drawn separately.

\section{Conclusions}

In summary, we have calculated the electronic structure of electrons confined in a Gaussian QD by using the UHF method in its Pople and Nesbet form. We gave a systematic procedure for constructing the basis set for the self-consistent calculations with a given precision from the one-electron problem. The orbital energies provide an insight of the occupancy of the levels and show the fulfillment of the Hund rule in agreement with previous results obtained with other models $[11,12]$.

Since the Gaussian potential has finite depth, binding and dissociation processes can occur. The criterion for the stability of an $\mathrm{N}$-electron system was already discussed in Ref. [10] in terms of its energy $E_{0}^{(N)}$ as compared to $E_{0}^{(N-1)}$. The condition $E_{0}^{(N)}<E_{0}^{(N-1)}$ is equivalent, in UHF calculations, to the condition that the highest occupied molecular orbital $(\mathrm{HOMO})$ is bound, i.e., $\varepsilon_{\text {HOMO }}<0$; this is a consequence of Koopman's theorem [25]. If the electronelectron interaction can be neglected, the regions of the $\left(V_{0}, R\right)$ plane having $N$ bound electrons are delimited by the relation $V_{0} R^{2}=$ const. This result has been proven to be a widely valid feature resulting from the scaling properties of the confining potential.

Both the chemical potential and the charging energy show a shell structure with peaks of stability at $N=2,5$, and 8 , corresponding to filled and half-filled shells. Both magnitudes decrease as the confinement range increases. The methodology applied to single QDs in this work, can straightforwardly be transferred to the study of the electronic structure and properties of QDs arrays. It is also suitable for considering correlation effects with standard atomic methods. Work along such lines is currently in progress.

\section{Acknowledgements}

This work has been supported by CONICET (Argentina), Agencia Nacional de Promoción Científica y Tecnológica (ANPCyT, Argentina) and Universidad Nacional del Nordeste under Grants PICTO-204/07 and PI-112/07.

\section{References}

[1] P. Harrison, Quantum wells, wires and dots (Wiley, UK, 2005)

[2] P. Michler, Single Quantum Dots, Fundamentals, Applications, and New Concepts (Springer-Verlag, Berlin-Heidelberg, 2003)

[3] R. Hanson, L. P. Kouwenhoven, J. R. Petta, S. Tarucha, L. M. K. Vandersypen, Rev. Mod. Phys. 79, 001217 (2007)

[4] D. D. Awschalom, M. Flatte, Nat. Phys. 3, 153 (2007)

[5] M. Atatüre et al., Science 312, 551 (2006)

[6] M. Bayer et al., Science 291, 451 (2001)

[7] S. M. Reimann, M. Manninen, Rev. Mod. Phys. 74, 1283 (2002)

[8] A. P. Alivisatos, J. Phys. Chem. 100, 13226 (1996)

[9] W. Kohn, Phys. Rev. 123, 1242 (1961)

[10] B. Szafran, J. Adamowski, S. Bednarek, Physica E 4, 1 (1999)

[11] S. Bednarek, B. Szafran, J. Adamowski, Phys. Rev. B 59, 13036 (1999)

[12] C. F. Destefani, J. D. M. Vianna, G. E. Marques, Semicond. Sci. Technol. 19, L90 (2004)

[13] V. Ranjan, R. K. Pandey, M. K. Harbola, V. A. Singh, Phys. Rev. 65, 045311 (2002)

[14] S. De Filippo, M. Salerno, Phys. Rev. B 62, 4230 (2000)

[15] J. Adamowski, M. Sobkowicz, B. Szafran, S. Bednarek, Phys. Rev. B 62, 4234 (2000), Erratum: Phys. Rev. B 62, 13233 (2000)

[16] W. Xie, Solid State Comm. 127, 401 (2003)

[17] B. Boyacioglu, M. Saglam, A. Chatterjee, J. Phys.: Condens. Matter 19, 456217 (2007)

[18] O. Ciftja, J. Computer-Aided Mater. Des. 14, 37 (2007)

[19] N. Bessis, G. Bessis, B. Joulakian, J. Phys. A: Math. Gen. 15, 3679 (1982)

[20] C. S. Lai, J. Phys. A: Math. Gen. 16, L181 (1983)

[21] M. Cohen, J. Phys. A: Math. Gen. 17, L101 (1984)

[22] A. Chatterjee, J. Phys. A: Math. Gen. 18, 2403 (1985)

[23] O. Gunawan, H. S. Djie, B. S. Ooi, Phys. Rev. B 71, 205319 (2005)

[24] P. Fulde, Electron Correlations in Molecules and solids (Springer-Verlag Berlin, 1995)

[25] A. Szabo, Ostlund, Modern Quantum Chemistry 
(Macmillan Publishing, New York, 1982)

[26] M. Tadic, F. M. Peeters, K. L. Janssens, Phys. Rev. B 65, 165333 (2002)

[27] E. R. Davidson, D. Feller, Chem. Rev. 86 681, (1986)

[28] J. M. Thijssen, Computational Physics (Cambridge University Press, Cambridge, 1999) 